\title{
Radial endobronchial ultrasound with a guide sheath for diagnosis of peripheral cavitary lung lesions: a retrospective study
}

\author{
Manabu Hayama1*, Norio Okamoto', Hidekazu Suzuki', Motohiro Tamiya', Takayuki Shiroyama', Ayako Tanaka', \\ Takuji Nishida', Takashi Nishihara', Nobuko Uehara', Naoko Morishita', Kunimitsu Kawahara² \\ and Tomonori Hirashima'
}

\begin{abstract}
Background: Radial endobronchial ultrasound with a guide sheath (EBUS-GS) has improved the diagnostic outcomes of peripheral lung lesions. However, to our knowledge, reports on the use of EBUS-GS for diagnosis of cavitary lesions are unavailable. Therefore, this study aimed to assess the effectiveness and safety of EBUS-GS for diagnosis of peripheral cavitary lung lesions (PCLLs).

Methods: This study was a single-institution retrospective review of PCLLS examined by using EBUS-GS between July 2013 and October 2015. The diagnostic results of different EBUS-GS samples, including cytologic, histopathologic, and microbiologic samples, were analysed separately.

Results: Of 696 radial EBUS procedures performed during the study period, 50 were performed for examination of PCLLs. The overall diagnostic yield for EBUS-GS was 80 \% (40/50). Regarding 27 malignant lesions, the diagnostic yields for cytologic and histopathologic samples were $63.0 \%$ (17/27) and 74.1\% (20/27), respectively. Regarding 23 benign lesions, the diagnostic yields for histopathologic and microbiologic samples were $69.6 \%(16 / 23)$ and $47.8 \%$ (11/23), respectively. Uni- and multivariate analyses indicated that the EBUS probe being within the lesion was the only factor significantly associated with increased diagnostic yield (odds ratio, $7.04 ; P=0.03$ ). Although pulmonary infection occurred after the procedure in 1 patient $(2.0 \%)$, no other complications, including pneumothorax or significant haemorrhage, were reported.
\end{abstract}

Conclusion: EBUS-GS was found to be an effective and safe procedure for diagnosis of PCLLS.

Keywords: Radial endobronchial ultrasound, Endobronchial ultrasound with a guide sheath, Lung cancer, Cavity, peripheral lung lesion, Transbronchial biopsy, Bronchoscopy

\section{Background}

A pulmonary cavity is defined as a gas-filled area within a pulmonary consolidation, mass, or nodule that may or may not contain a fluid level and is surrounded by a wall, usually of varied thickness [1]. Various malignant and benign diseases can present as cavitary lung lesions. Because findings on chest radiography and computed tomography (CT) often overlap between malignant and benign cavitary

\footnotetext{
* Correspondence: manabuhayama@gmail.com

'Department of Thoracic Malignancy, Osaka Prefectural Medical Center for Respiratory and Allergic Diseases, 3-7-1, Habikino, Habikino City, Osaka 583-8588, Japan

Full list of author information is available at the end of the article
}

lesions, minimally invasive specimen collection is often necessary for accurate diagnosis [2]. However, sufficient diagnostic biopsy samples may not be obtained due to the limited target area of the cavity wall and the surrounding reactive normal tissue [3].

According to the guidelines of the American College of Chest Physicians, the sensitivity of transbronchial biopsy (TBB) for diagnosis of peripheral lung lesions is $63 \%$ for lesions $>20 \mathrm{~mm}$, and only $34 \%$ for lesions $<20 \mathrm{~mm}$ [4]. On the other hand, the pooled sensitivity of transthoracic needle aspiration (TTNA) for diagnosis of peripheral lung cancer is $90 \%$, making it the preferred method for preoperative diagnosis [4]. However, TTNA carries a high 
risk of complications, such as pneumothorax, air embolism, and tumour seeding $[5,6]$. With regard to bronchoscopy for diagnosis of peripheral lung lesions, a recent metaanalysis reported a diagnostic yield of $70 \%$ with use of electromagnetic navigation bronchoscopy, radial endobronchial ultrasound (R-EBUS) with or without a guide sheath, virtual bronchoscopic navigation (VBN), and ultrathin bronchoscopes; among these, the pooled diagnostic yield was highest with use of a guide sheath [7]. Therefore, because of its safety profile and sufficient diagnostic yield, R-EBUS with a guide sheath (EBUS-GS) is now becoming a common procedure for examination of peripheral lung lesions [8].

However, reports on the use of R-EBUS for diagnosis of cavitary lung lesions with a relatively high probability of infectious disease are limited [9]. Because it is unknown whether a lung lesion is malignant or benign before diagnostic bronchoscopy, malignant and benign lesions usually are examined by using the same procedure [10]. The aim of this study was to assess the effectiveness and safety of EBUS-GS for diagnosis of peripheral cavitary lung lesions (PCLLs).

\section{Methods}

\section{Study approval}

This study was a retrospective review of a prospectively maintained database and medical records at our institution. The Institutional Review Board of Osaka Prefectural Medical Center for Respiratory and Allergic Diseases approved this study without the need to obtain informed consent. Written informed consent for bronchoscopy was obtained from all eligible patients.

\section{Patient selection}

Patient information (age, sex, type of procedure) and lesion characteristics were collected prospectively in the database before diagnostic bronchoscopy. Lesion characteristics, including type (solid, part-solid, pure ground-glass opacity [GGO], cavitary, or other [i.e., atelectasis, diffuse or lobar consolidation]), maximum diameter, target bronchus, and presence of bronchus sign, were evaluated on thin-section chest CT images during regular team discussions that included board-certified bronchoscopists, pulmonologists, and medical oncologists. VBN (LungPoint, Bronchus Ltd.) was used when the target bronchus was small and difficult to trace [11].

PCLLs examined by using EBUS-GS at our institution between July 2013 and October 2015 were included in this study. Cases in which ultrathin bronchoscopes (BFXP260, Olympus Ltd.) were additionally used were excluded. A PCLL was defined as a bronchoscopically invisible lung lesion that had an internal gas-filled area and was surrounded by pulmonary parenchyma $[1,8]$. Maximum cavity wall thickness and minimum distance from the costal pleura were additionally assessed on chest CT transverse images.

\section{EBUS-GS procedure}

All EBUS-GS procedures were performed by expert bronchoscopists or trainees with sufficient experience with conventional bronchoscopy and EBUS-GS assistance. During all the procedures, the trainees were supervised by the experts.

EBUS-GS procedures were carried out by using one of the following bronchoscopes (Olympus Ltd.): BF-1T260, BF-P260, BF-F260, or BF-Y0053, a new middle-range bronchoscope with a 5.1-mm outer diameter and a 2.6mm working channel [12]. A guide sheath kit (K-202 or K-204, Olympus Ltd.) was used in combination with an R-EBUS probe (UM-S20-17S, Olympus Ltd.). All bronchoscopies were performed via an oral route under local anaesthesia with intravenous midazolam for mild sedation.

Upon insertion of the scope to reach the target bronchus, the guide sheath together with the R-EBUS probe was inserted through the working channel and advanced under fluoroscopic guidance. EBUS images before transbronchial sampling were categorized into 3 patterns: 'within' (the probe was located in a bronchus that was inside the lesion), 'adjacent to' (the probe was located in a bronchus that ran alongside the lesion), or 'invisible' (the probe was not able to reach the lesion) [8]. After locating the lesion, the probe was removed while the guide sheath was kept in place for subsequent sampling, also under fluoroscopic guidance. Transbronchial needle aspiration (TBNA) with or without a guide sheath was additionally performed when the operator deemed that the sample amount was insufficient $[11,13]$.

Brush and needle smears and tissue stumps on glass slides were sent for cytologic examination. Tissue samples obtained by using forceps were sent for histopathologic examination. After each sampling, the brush, needles, and forceps were rinsed with $5 \mathrm{~mL}$ normal saline (device wash) [14]. After all the samplings, the guide sheath was removed and the lumen was flushed with the device wash liquid. Then, the device wash sample was divided into 2 test tubes, which were sent for cytologic and microbiologic examinations, respectively (Fig. 1). Routine microbiologic examination comprised Gram stain, acid-fast bacilli smear, polymerase chain reaction of Mycobacterium tuberculosis (TB), and cultures including TB. Although additional bronchial wash was performed in 2 patients, those results were not assessed in this study.

\section{Diagnostic criteria}

Final diagnosis was determined based on either one or a combination of the results of EBUS-GS, EBUS-TBNA, 


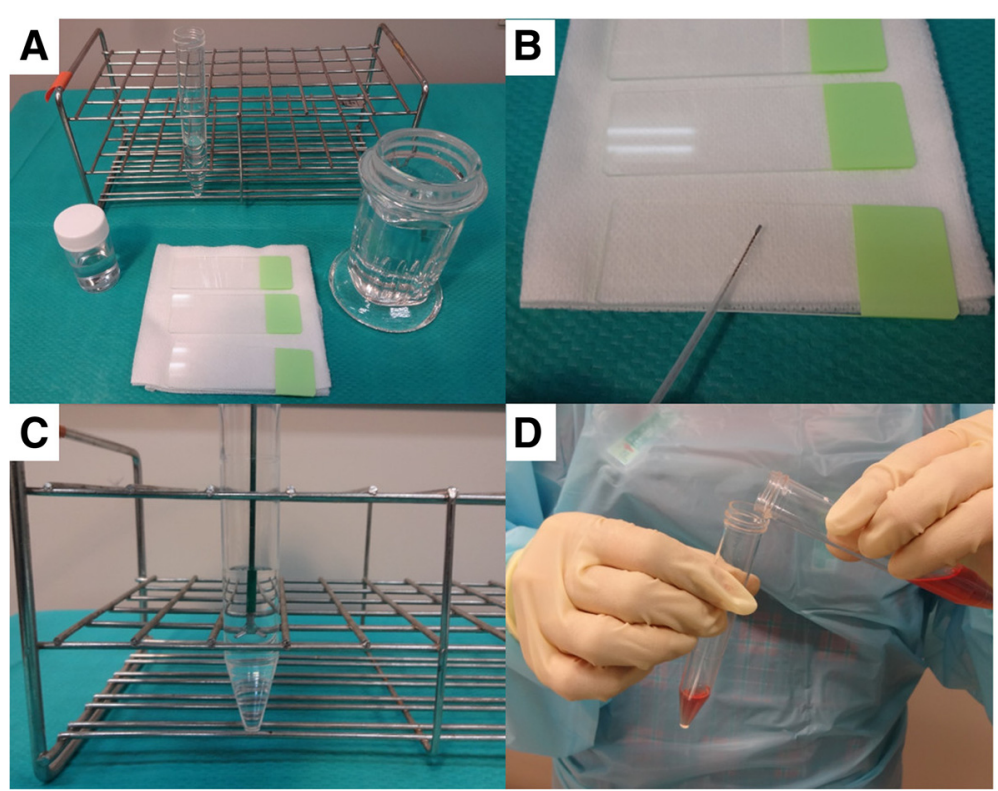

Fig. 1 Specimen collection set for endobronchial ultrasound with a guide sheath (EBUS-GS). (a) A specimen collection set for EBUS-GS routinely consists of $10 \%$ formalin (lower left), glass slides (lower centre), $95 \%$ alcohol (lower right), and 5 mL normal saline (upper). (b) Brush and needle smears and tissue stumps on glass slides are sent for cytologic examination. (c) After each sampling, the brush, needles, and forceps are rinsed with $5 \mathrm{~mL}$ normal saline (device wash). (d) The device wash sample is divided into 2 test tubes, which are sent for cytologic and microbiologic examinations, respectively

surgery, or clinical follow-up for at least 3 months. A lesion was designated as inflammation if it showed no significant pathologic or clinical findings, and it decreased in size on follow-up CT.

For malignant lesions, class IV/V results on cytology or specific malignant findings on histopathology were considered as diagnostic. For benign lesions, specific benign findings on histopathology (e.g. necrotizing epithelioid granuloma, acute-angle branching hyphae, or inflammation) or positive cultures on microbiology were considered as diagnostic. Histopathologic results were reviewed by 2 investigators (MH and KK). Overall, EBUS-GS was considered as diagnostic when at least 1 of the cytologic, histopathologic, or microbiologic results was diagnostic.

\section{Complications of EBUS-GS}

All bronchoscopies were performed in inpatient settings. After every procedure, chest radiography was performed and oral antibiotics (cefcapene pivoxil $300 \mathrm{mg} /$ day for 3 days) were administered. A major complication was defined as an event that necessitated premature termination of the procedure or a symptomatic postprocedure sequela, including pneumothorax, haemorrhage, infection, air embolism, or other untoward life-threatening outcome [8].

\section{Statistical analysis}

Descriptive statistics are presented as frequency, percentage, and median (range). Univariate analyses were performed by using Fisher's exact test for categorical data. Multivariate logistic regression analysis was performed to determine the factors associated with increased diagnostic yield. Variables for the multivariate analysis model were chosen from the results of the univariate analyses and experimentally predicted factors, such as lesion size and bronchus sign. A 2-tailed $P$ value of $<0.05$ was considered to indicate statistical significance. All statistical analyses were performed by using EZR (Saitama Medical Center, Jichi Medical University, Saitama, Japan), a graphical user interface for $\mathrm{R}$ (The $\mathrm{R}$ foundation for Statistical Computing, Vienna, Austria) [15].

\section{Results}

Among 696 R-EBUS procedures performed between July 2013 and October 2015 at our institution, 50 PCLLs examined by using EBUS-GS were included in this study (Fig. 2). A summary of the baseline characteristics of the 50 patients is shown in Table 1. The study population had a median age of 67 years and mostly consisted of male patients. Median lesion size, cavity wall thickness, and distance from the costal pleura were $33.5,11$, and $0 \mathrm{~mm}$, respectively. Most lesions were located in the lower lobe (54\%), and bronchus sign was positive in the majority of cases (82\%). VBN was used for route planning in 27 patients (54\%).

Details of the final diagnoses are shown in Table 2. Among 27 malignant lesions, 21 were successfully 


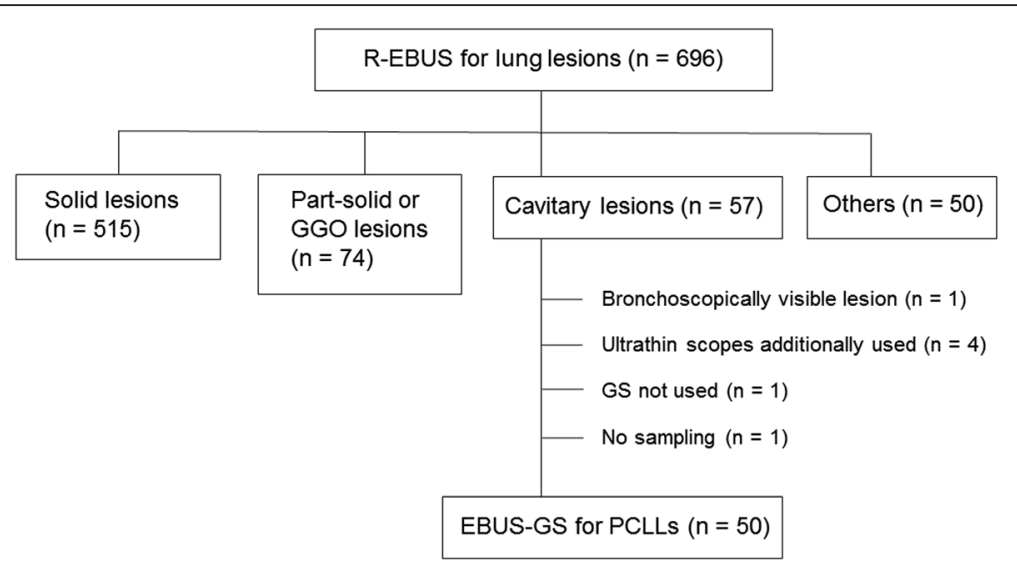

Fig. 2 Study diagram. Abbreviations: EBUS-GS endobronchial ultrasound with a guide sheath, GGO ground-glass opacity, GS guide sheath, PCLL peripheral cavitary lung lesion, R-EBUS radial endobronchial ultrasound

diagnosed by using EBUS-GS (diagnostic yield, $77.8 \%$ ), 4 were diagnosed according to other examinations after EBUS-GS (EBUS-TBNA in 1 lung adenocarcinoma, surgery in 2 lung adenocarcinomas and 1 lung squamous carcinoma), and the remaining 2 were clinically diagnosed as lung cancer according to more than 6 months of CT follow-up and multidisciplinary team discussions. Among 23 benign

Table 1 Baseline characteristics of patients with peripheral cavitary lung lesions

\begin{tabular}{lc}
\hline Variable & All $(n=50)$ \\
\hline Age, median (range), year & $67(45-86)$ \\
Sex, $n$ (\%) & \\
$\quad$ Male & $32(64)$ \\
Female & $18(36)$ \\
Lesion size, median (range), mm & $33.5(16-100)$ \\
Cavity wall thickness, median (range), mm & $11(3-64)$ \\
Distance from costal pleura, median (range), mm & $0(0-22)$ \\
Lobar location, $n$ (\%) & \\
$\quad$ Upper & $21(42)$ \\
Middle & $2(4)$ \\
Lower & $27(54)$ \\
Bronchus sign, $n$ (\%) & \\
Positive & $41(82)$ \\
Negative & $9(18)$ \\
VBN, $n$ (\%) & \\
Yes & $27(54)$ \\
No & $23(46)$ \\
EBUS, $n$ (\%) & \\
Within & \\
Adjacent to & $18(36)$ \\
Invisible &
\end{tabular}

Abbreviations: EBUS endobronchial ultrasound, VBN virtual bronchoscopic navigation lesions, 19 were successfully diagnosed by using EBUS-GS (diagnostic yield, $82.6 \%$ ), 3 were diagnosed according to other examinations after EBUS-GS (surgery in 1 nontuberculous mycobacteria [NTM], sputum culture in 2 NTMs), and the remaining 1 was clinically diagnosed as rheumatoid nodule based on its marked decrease in size by immunosuppressive therapy for rheumatoid arthritis. Overall, sensitivity, specificity, positive predictive value and negative predictive value for diagnosing malignancy were $77.8,100$, 100 and $79.3 \%$, respectively.

The diagnostic yields for cytologic, histopathologic and microbiologic samples also are shown in Table 2. For 27 malignant lesions, 17 (63.0 \%) cytologic and 20 (74.1\%) histopathologic samples were diagnostic. With respect to histologic type of lung cancer, squamous cell carcinoma was more diagnosable by histopathology than adenocarcinoma (86.7 vs $70 \%$ ). For 23 benign lesions, 16 (69.6\%) histopathologic and 11 (47.8\%) microbiologic samples were diagnostic. For 15 lesions with mycobacterial infection (TB in 3, NTM in 12), the sensitivity of EBUS-GS was $80 \%(12 / 15)$, and $9(60 \%)$ and 11 (73.3\%) lesions were successfully diagnosed by using histopathologic and microbiologic samples, respectively. All of the other infectious diseases, including 4 lung abscesses and 1 Aspergillosis, were successfully diagnosed by histopathologic samples only.

The overall diagnostic yield for EBUS-GS was $80 \%$ (40/50). Univariate analyses indicated that PCLLs in which the EBUS probe could be placed within the lesion had a significantly higher diagnostic yield compared with when the EBUS probe was adjacent to the lesion or invisible ( 92.3 vs. $66.7 \%, P=0.03)$. Multivariate logistic regression analyses using the possible cofounders of lesion size, bronchus sign and EBUS probe 'within' also revealed that the only significant factor affecting diagnostic yield was EBUS probe 'within' (odds ratio, 7.04; $P=0.03$ ) (Table 3). Among the 50 procedures, pulmonary 
Table 2 Final diagnoses and diagnostic yields according to sample category

\begin{tabular}{|c|c|c|c|c|c|}
\hline Final diagnosis & $\begin{array}{l}\text { All } \\
(n=50)\end{array}$ & $\begin{array}{l}\text { Diagnosed by EBUS- } \\
\text { GS, } n(\%)\end{array}$ & $\begin{array}{l}\text { Diagnosed by cytologic } \\
\text { sample, } n(\%)\end{array}$ & $\begin{array}{l}\text { Diagnosed by histologic } \\
\text { sample, } n(\%)\end{array}$ & $\begin{array}{l}\text { Diagnosed by microbiologic } \\
\text { sample, } n(\%)\end{array}$ \\
\hline Lung cancer & 27 & $21(77.8)$ & $17(63.0)$ & $20(74.1)$ & $0(0)$ \\
\hline Adenocarcinoma & 10 & $7(70)$ & $6(60)$ & $7(70)$ & $0(0)$ \\
\hline $\begin{array}{l}\text { Squamous cell } \\
\text { carcinoma }\end{array}$ & 15 & $14(93.3)$ & $11(73.3)$ & $13(86.7)$ & $0(0)$ \\
\hline $\begin{array}{l}\text { Clinically } \\
\text { diagnosed }\end{array}$ & 2 & $0(0)$ & $0(0)$ & $0(0)$ & $0(0)$ \\
\hline Benign & 23 & 19 (82.6) & $0(0)$ & $16(69.6)$ & $11(47.8)$ \\
\hline TB & 3 & $3(100)$ & $0(0)$ & $3(100)$ & $3(100)$ \\
\hline NTM & 12 & $9(75)$ & $0(0)$ & $6(50)$ & $8(66.7)$ \\
\hline Lung abscess & 4 & $4(100)$ & $0(0)$ & $4(100)$ & $0(0)$ \\
\hline Aspergillosis & 1 & $1(100)$ & $0(0)$ & $1(100)$ & $0(0)$ \\
\hline $\begin{array}{l}\text { Rheumatoid } \\
\text { nodule }\end{array}$ & 1 & $0(0)$ & $0(0)$ & $0(0)$ & $0(0)$ \\
\hline Inflammation & 2 & $2(100)$ & $0(0)$ & $2(100)$ & $0(0)$ \\
\hline
\end{tabular}

Abbreviations: EBUS-GS endobronchial ultrasound with a guide sheath, NTM nontuberculous mycobacteria, TB Mycobacterium tuberculosis

infection occurred in 1 patient (2.0\%). In this case, although fever and increased inflammatory reaction occurred after EBUS-GS, such symptoms were resolved by intravenous antibiotic therapy. No other major complications, including pneumothorax or significant haemorrhage that necessitated premature termination of the procedure, were reported.

A representative case is shown in Fig. 3. The EBUS image before sampling was 'adjacent to' the lesion, which showed the thin wall of the lesion. With the guide sheath located just before the cavity wall, samples were obtained by using a brush and forceps. Cytologic and histologic specimens showed adenocarcinoma.

\section{Discussion}

Although EBUS-GS has improved the diagnostic yield for peripheral lung lesions, reports on the use of EBUSGS specifically for cavitary lesions are unavailable. To the best of our knowledge, this is the first report on the efficacy and safety of EBUS-GS for diagnosis of PCLLs. In addition, the utility of the practical sampling method of EBUS-GS for malignant and benign PCLLs was carefully evaluated in this study. Two important clinical observations were made. First, EBUS-GS had a high diagnostic yield $(80 \%)$ for PCLLs with a low risk of significant complications (2.0\%). Second, the EBUS-GS sampling method combined with cytologic, histopathologic, and microbiologic samples was effective for diagnosis of both benign and malignant PCLLs.

A cavity is the result of such pathologic processes as necrosis, cystic dilation of pulmonary structures, and displacement of lung tissue by cystic structures [16]. In addition, malignant lesions can become hollowed out due to treatment-related necrosis, internal cyst formation, or internal desquamation of tumour cells [17]. Active infectious and malignant diseases require a prompt and accurate diagnosis to minimize adverse outcomes and apply appropriate management. Although thin-section chest CT findings, such as cavity wall thickness, are helpful for diagnosis of cavitary lesions, sufficient pathologic or microbiologic samples are often necessary to ensure correct diagnosis [16]. TTNA is a useful nonsurgical method for examination of peripheral lung lesions, but its risk of complication is generally higher than TBB, and thus, it may have concerns about safety for cavitary lesions because of internal necrosis and inflammatory reactions $[18,19]$. Actually, Zhuang et al. reported that although TTNA had an accuracy of $96.1 \%$ for diagnosis of 102 cavitary lung lesions, complications included pneumothorax $(8.8 \%)$, alveolar haemorrhage (13.7\%), and hemoptysis (1\%) [20].

Currently, EBUS-GS is becoming a standard procedure for diagnosis of peripheral lung lesions based on its useful and safe profile. Therefore, confirmation of its diagnostic utility for PCLLs is also demanded in clinical practice. In the present study, we first reported a high diagnostic yield (80 \%) for EBUS-GS for PCLLs. This result is comparable to the diagnostic yield for R-EBUS for peripheral lung lesions reported in recent studies. Therefore, EBUS-GS can be considered as a standard procedure for diagnosis of peripheral lung lesions and PCLLs $[9,21-23]$. Moreover, we demonstrated that the diagnostic yield was better when the R-EBUS probe was within the lesion, which has been similarly shown in previous studies [24-26]. However, in the present study, no other important factors, such as bronchus sign, lesion size, or cavity wall thickness, were found to be associated with increased diagnostic yield, despite the small sample size. Especially in larger lesions, the inflammatory reaction 
Table 3 Factors affecting diagnostic yield of EBUS-GS for peripheral cavitary lung lesions

\begin{tabular}{|c|c|c|c|c|}
\hline \multirow[t]{2}{*}{ Variable } & \multicolumn{2}{|l|}{ Univariate analysis } & \multicolumn{2}{|c|}{$\underline{\text { Multivariate analysis }}$} \\
\hline & Diagnostic yield, (\%) & $P$ value & $\mathrm{OR}(95 \% \mathrm{Cl})$ & $P$ value \\
\hline \multicolumn{5}{|l|}{ Age, year } \\
\hline$\geq 70$ & $17 / 20(85)$ & 0.72 & - & \\
\hline$<70$ & $23 / 30(76.7)$ & & & \\
\hline \multicolumn{5}{|l|}{ Sex } \\
\hline Male & 26/32 (81.3) & 1 & - & \\
\hline Female & 14/18 (77.8) & & & \\
\hline \multicolumn{5}{|l|}{ Lesion size, mm } \\
\hline$>30$ & 25/31 (80.6) & 1 & $1.19(0.24-5.95)$ & 0.83 \\
\hline$\leq 30$ & 15/19 (78.9) & & & \\
\hline \multicolumn{5}{|l|}{ Cavity wall thickness, mm } \\
\hline$>10$ & $22 / 27(81.5)$ & 1 & - & \\
\hline$\leq 10$ & 18/23 (78.3) & & & \\
\hline \multicolumn{5}{|c|}{ Distance from costal pleura, mm } \\
\hline$>10$ & 10/11 (90.9) & 0.42 & - & \\
\hline$\leq 10$ & $30 / 39(76.9)$ & & & \\
\hline \multicolumn{5}{|l|}{ Lobar location } \\
\hline Lower & 21/27 (77.8) & 0.74 & - & \\
\hline Upper/middle & 19/23 (82.6) & & & \\
\hline \multicolumn{5}{|l|}{ Bronchus sign } \\
\hline Positive & $32 / 41(78.0)$ & 0.67 & $0.26(0.02-2.90)$ & 0.27 \\
\hline Negative & 8/9 (88.9) & & & \\
\hline \multicolumn{5}{|l|}{ VBN } \\
\hline Yes & $22 / 27(81.5)$ & 1 & - & \\
\hline No & 18/23 (78.3) & & & \\
\hline \multicolumn{5}{|l|}{ EBUS image } \\
\hline Within & $24 / 26(92.3)$ & 0.03 & $7.04(1.27-38.90)$ & 0.03 \\
\hline Adjacent to/invisible & $16 / 24(66.7)$ & & & \\
\hline
\end{tabular}

Abbreviations: Cl confidence interval, EBUS endobronchial ultrasound, EBUS-GS endobronchial ultrasound with a guide sheath, OR odds ratio, VBN virtual bronchoscopic navigation

around the cavity wall might interfere with obtaining diagnostic samples even if bronchus sign is positive. A previous study on the use of TTNA for diagnosis of cavitary lung lesions reported that more nondiagnostic samples were obtained from lesions with thin walls [20]. Repeated biopsies at precise locations might contribute to accurate diagnosis even for PCLLs with thin walls, as shown in the representative case.

The complication rate in the present study was $2.0 \%$ (pulmonary infection), which is lower than that reported in previous studies on the use of TTNA for diagnosis of cavitary lung lesions $[3,20]$. There was no case of pneumothorax or significant haemorrhage, which are major complications of TBB for peripheral lung lesions. Precise localisation by R-EBUS and the tamponade effect of the guide sheath might reduce the risk of complications, even for PCLLs. Although TBB using R-EBUS has been reported as a safe procedure for diagnosis of peripheral lung lesions, a previous study showed that among 965 peripheral lung lesions examined by using EBUS-GS, pulmonary infection occurred in 5 patients (0.5\%), 2 of which had PCLLs [8]. Therefore, although EBUS-GS was found to be safe for diagnosis of PCLLs in the present study, we should be mindful of the risk of pulmonary infection.

This study also demonstrated that the EBUS-GS sampling method combined with cytologic, histopathologic, and microbiologic samples was effective for diagnosis of both benign and malignant PCLLs. In the present study, diagnostic tissue samples were obtained by using TBB for $74.1 \%$ of malignant lesions and $69.6 \%$ of benign lesions. Recently, histopathologic diagnosis of lung cancer is becoming important for definitive treatment including chemotherapy and tyrosine kinase inhibitors [27]. 


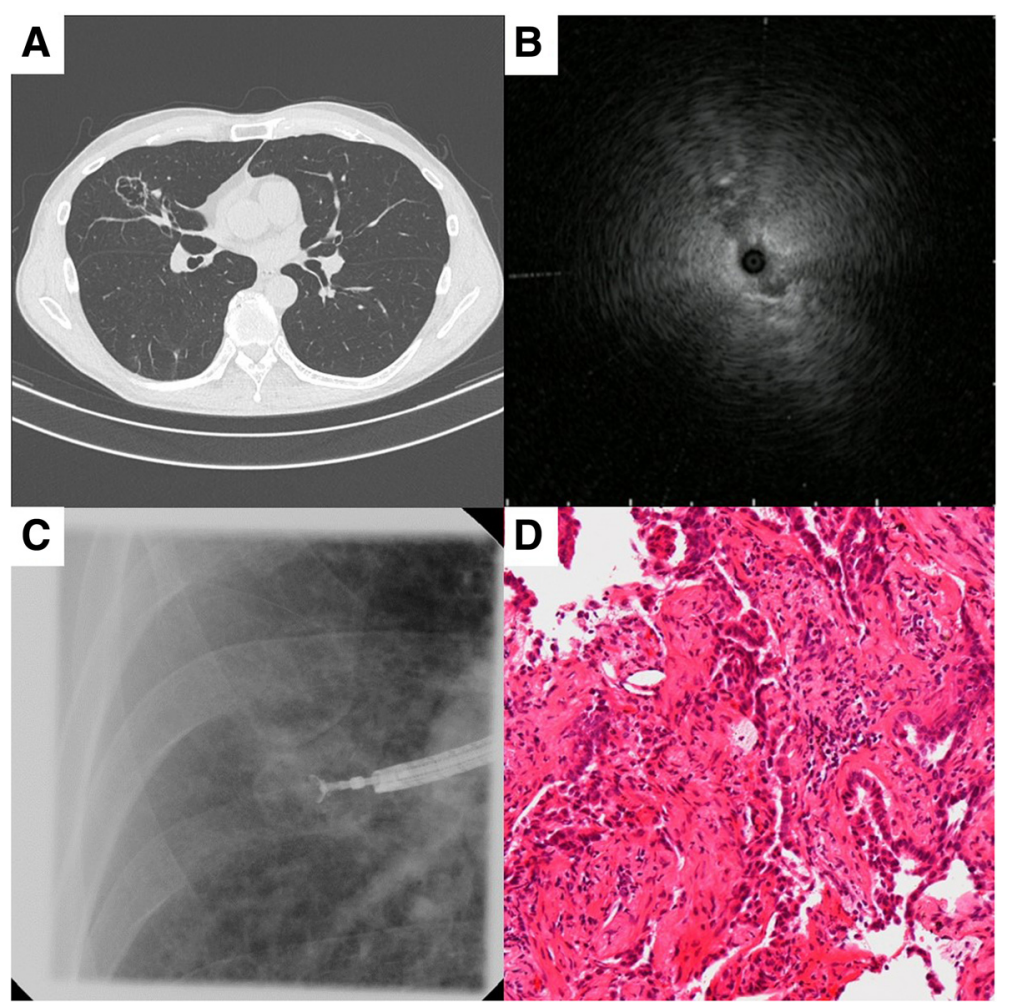

Fig. 3 Case of a 64-year-old man with an abnormal shadow in the right middle lobe. (a) Computed tomography scan, showing a 32-mm peripheral cavitary lung lesion with a thin wall $(3 \mathrm{~mm}$ ). (b) Endobronchial ultrasound image is 'adjacent to' the lesion. (c) Fluoroscopic image during transbronchial biopsy through a guide sheath. (d) Histologic specimen obtained by using forceps, showing adenocarcinoma (hematoxylin and eosin stain, magnification $\times 200$ )

Moreover, because cavitary lung cancer has a worse prognosis than non-cavitary lung cancer, successful histopathologic diagnosis using a safe technique is demanded [28]. This should also be applied to benign cavitary lesions. Chan et al. recently reported that necrotizing granulomatous inflammation was demonstrated by using EBUS-GS in 10 of 22 patients with pulmonary $\mathrm{TB}$, and that histopathologic diagnosis of TB might contribute to prompt treatment [9]. Certainly, histopathologic diagnosis is necessary for appropriate treatment not only for malignant lesions but also for benign lesions. Even for PCLLs, EBUS-GS has the advantages of locating lesions accurately, controlling bleeding, and obtaining adequate tissue samples.

In the present study, microbiologic samples demonstrated a diagnostic yield of $47.8 \%$ for benign diseases; the diagnostic yield was particularly high for mycobacterium infection, which is the main cause of a cavitary lung lesion (100 \% in TB, $66.7 \%$ in NTM). Microbiology results were negative for infectious cases with lung abscess or Aspergillosis, though anaerobic or Aspergillus culture was not performed routinely. Because the culture sensitivities of these diseases using bronchoscopy samples are not satisfactory $[29,30]$, routine bronchial wash or bronchoalveolar lavage after removing the guide sheath may not be necessary. Instead, obtaining adequate tissue samples can contribute to definitive treatment. Although the guide sheath has to be introduced into the lesion for adequate tissue sampling, the R-EBUS probe was located within the lesion in only 26 patients (52\%) in the present study, despite a positive bronchus sign in the majority of cases (82\%). Although this may be due to a lack of skills in the trainees, additional TBNA may be important for improved diagnosis of PCLLs, especially when they are not located by using REBUS [13, 31-33].

The present study has several limitations. First, this was a retrospective, nonrandomised, single-institution study with a small sample size. Second, the procedures were not performed by the same operator. Thus, it remains unclear whether the results can be generalized to other institutions.

\section{Conclusions}

In conclusion, we presented the first report on EBUS-GS for PCLLs based on our clinical experience. Our results indicate that EBUS-GS is a safe and effective procedure for diagnosis of PCLLs. Sufficient cytologic, histopathologic, 
and microbiologic samples can be obtained for both benign and malignant PCLLs. Further multicenter prospective studies are recommended in the future.

\section{Ethics approval and consent to participate}

The Institutional Review Board of Osaka Prefectural Medical Center for Respiratory and Allergic Diseases approved this study without the need to obtain informed consent.

\section{Availability of data and materials}

The dataset supporting the conclusions of this article is presented within the article. The detailed clinical data set is not publically available to protect research subject privacy and confidentiality.

\section{Abbreviations}

CT: computed tomography; EBUS: endobronchial ultrasound; EBUSGS: endobronchial ultrasound with a guide sheath; GGO: ground-glass opacity; NTM: nontuberculous mycobacteria; PCLL: peripheral cavitary lung lesion; R-EBUS: radial endobronchial ultrasound; TB: Mycobacterium tuberculosis; TBB: transbronchial biopsy; TBNA: transbronchial needle aspiration; TTNA: transthoracic needle aspiration; VBN: virtual bronchoscopic navigation.

\section{Competing interests}

The authors declare that they have no competing interests.

\section{Authors' contributions}

$\mathrm{MH}$ designed the overall study and performed the statistical analysis. All authors substantially contributed to the acquisition, interpretation, and consolidation of data. All authors wrote the manuscript and approved the final version.

\section{Acknowledgments}

We thank the staff members of the Department of Thoracic Malignancy at Osaka Prefectural Medical Center for Respiratory and Allergic Diseases for their efforts in data collection.

\section{Funding}

None.

\section{Author details}

'Department of Thoracic Malignancy, Osaka Prefectural Medical Center for Respiratory and Allergic Diseases, 3-7-1, Habikino, Habikino City, Osaka 583-8588, Japan. ²Department of Pathology, Osaka Prefectural Medical Center for Respiratory and Allergic Diseases, Osaka, Japan.

Received: 22 February 2016 Accepted: 5 May 2016

Published online: 11 May 2016

\section{References}

1. Tuddenham WJ. Glossary of terms for thoracic radiology: recommendations of the Nomenclature Committee of the Fleischner Society. AJR Am J Roentgenol. 1984;143:509-17.

2. Honda O, Tsubamoto M, Inoue A, Johkoh T, Tomiyama N, Hamada S, et al. Pulmonary cavitary nodules on computed tomography: differentiation of malignancy and benignancy. J Comput Assist Tomogr. 2007;31:943-9.

3. Belet U, Findik S, Ozmen Z, Atici AG, Akan H. Percutaneous cavitary lavage in the diagnosis of pulmonary cavities. J Thorac Dis. 2013:5:440-5.

4. Rivera MP, Mehta AC, Wahidi MM. Establishing the diagnosis of lung cancer: diagnosis and management of lung cancer, 3rd ed: American College of Chest Physicians evidence-based clinical practice guidelines. Chest. 2013; 143(5 Suppl):e142S-65.

5. Tomiyama N, Yasuhara Y, Nakajima Y, Adachi S, Arai Y, Kusumoto M, et al. $C T$-guided needle biopsy of lung lesions: a survey of severe complication based on 9783 biopsies in Japan. Eur J Radiol. 2006;59:60-4
6. Wiener RS, Schwartz LM, Woloshin S, Welch HG. Population-based risk for complications after transthoracic needle lung biopsy of a pulmonary nodule: an analysis of discharge records. Ann Intern Med. 2011;155:137-44.

7. Wang Memoli JS, Nietert PJ, Silvestri GA. Meta-analysis of guided bronchoscopy for the evaluation of the pulmonary nodule. Chest. 2012;142:385-93.

8. Hayama M, Izumo T, Matsumoto Y, Chavez C, Tsuchida T, Sasada S. Complications with endobronchial ultrasound with a guide sheath for the diagnosis of peripheral pulmonary lesions. Respiration. 2015;90:129-35.

9. Chan A, Devanand A, Low SY, Koh MS. Radial endobronchial ultrasound in diagnosing peripheral lung lesions in a high tuberculosis setting. BMC Pulm Med. 2015;15:90

10. Shinagawa N, Nakano K, Asahina H, Kikuchi E, Ito T, Matsuno Y, et al. Endobronchial ultrasonography with a guide sheath in the diagnosis of benign peripheral diseases. Ann Thorac Surg. 2012;93:951-7.

11. Tamiya M, Okamoto N, Sasada S, Shiroyama T, Morishita N, Suzuki H, et al. Diagnostic yield of combined bronchoscopy and endobronchial ultrasonography, under LungPoint guidance for small peripheral pulmonary lesions. Respirology. 2013;18:834-9.

12. Sasada S, Izumo T, Chavez C, Matsumoto Y, Tsuchida T. A new middle-range diameter bronchoscope with large channel for transbronchial sampling of peripheral pulmonary lesions. Jpn J Clin Oncol. 2014;44:826-34.

13. Hayama M, Izumo T, Chavez C, Matsumoto $Y$, Tsuchida T, Sasada S. Additional transbronchial needle aspiration through a guide sheath (GSTBNA) for peripheral pulmonary lesions that cannot be detected by radial EBUS. Clin Respir J. 2015 Nov 25. [Epub ahead of print]

14. Izumo T, Sasada S, Chavez C, Matsumoto Y, Hayama M, Tsuchida T. The diagnostic value of histology and cytology samples during endobronchial ultrasound with a guide sheath. Jpn J Clin Oncol. 2015;45:362-6.

15. Kanda Y. Investigation of the freely available easy-to-use software 'EZR' for medical statistics. Bone Marrow Transplant. 2013;48:452-8.

16. Gadkowski LB, Stout JE. Cavitary pulmonary disease. Clin Microbiol Rev. 2008;21:305-33

17. Miura H, Taira O, Hiraguri S, Hagiwara M, Kato H. Cavitating adenocarcinoma of the lung. Ann Thorac Cardiovasc Surg. 1998:4:154-8.

18. Seo H, Cha SI, Shin KM, Lim J, Yoo SS, Lee J, et al. Focal necrotizing pneumonia is a distinct entity from lung abscess. Respirology. 2013;18: 1095-100.

19. Kim NR, Han J. Pathologic review of cystic and cavitary lung diseases. Korean J Pathol. 2012:46:407-14

20. Zhuang YP, Wang HY, Zhang J, Feng Y, Zhang L. Diagnostic accuracy and safety of CT-guided fine needle aspiration biopsy in cavitary pulmonary lesions. Eur J Radiol. 2013:82:182-6.

21. Oki M, Saka H, Ando M, Asano F, Kurimoto N, Morita K, et al. Ultrathin bronchoscopy with multimodal devices for peripheral pulmonary lesions. A randomized trial. Am J Respir Crit Care Med. 2015;192:468-76.

22. Steinfort DP, Bonney A, See K, Irving LB. Sequential multimodality bronchoscopic investigation of peripheral pulmonary lesions. Eur Respir J. 2016:47:607-14.

23. Matsumoto Y, Izumo T, Sasada S, Tsuchida T, Ohe Y. Diagnostic utility of endobronchial ultrasound with a guide sheath under the computed tomography workstation (ziostation) for small peripheral pulmonary lesions. Clin Respir J. 2015 June 15. [Epub ahead of print]

24. Chavez C, Sasada S, Izumo T, Watanabe J, Katsurada M, Matsumoto Y, et al. Endobronchial ultrasound with a guide sheath for small malignant pulmonary nodules: a retrospective comparison between central and peripheral locations. J Thorac Dis. 2015;7:596-602.

25. Tay JH, Irving L, Antippa P, Steinfort DP. Radial probe endobronchial ultrasound: factors influencing visualization yield of peripheral pulmonary lesions. Respirology. 2013;18:185-90.

26. Yamada N, Yamazaki K, Kurimoto N, Asahina H, Kikuchi E, Shinagawa N, et al. Factors related to diagnostic yield of transbronchial biopsy using endobronchial ultrasonography with a guide sheath in small peripheral pulmonary lesions. Chest. 2007;132:603-8.

27. Hirashima T, Suzuki H, Okamoto N, Morishita N, Yamadori T, Tamiya M, et al. Important factors for achieving survival of five years or more in non-small cell lung cancer patients with distant metastasis. Oncol Lett. 2014;8:327-34.

28. Watanabe Y, Kusumoto M, Yoshida A, Suzuki K, Asamura H, Tsuta K. Surgically resected solitary cavitary lung adenocarcinoma: association between clinical, pathologic, and radiologic findings and prognosis. Ann Thorac Surg. 2015;99:968-74. 
29. Ascioglu S, Rex JH, de Pauw B, Bennett JE, Bille J, Crokaert F, et al. Defining opportunistic invasive fungal infections in immunocompromised patients with cancer and hematopoietic stem cell transplants: an international consensus. Clin Infect Dis. 2002:34:7-14.

30. Hammond JM, Potgieter PD, Hanslo D, Scott H, Roditi D. The etiology and antimicrobial susceptibility patterns of microorganisms in acute community-acquired lung abscess. Chest. 1995;108:937-41.

31. Chao TY, Chien MT, Lie CH, Chung YH, Wang JL, Lin MC. Endobronchial ultrasonography-guided transbronchial needle aspiration increases the diagnostic yield of peripheral pulmonary lesions: a randomized trial. Chest. 2009;136:229-36.

32. Ost DE, Ernst A, Lei X, Kovitz KL, Benzaquen S, Diaz-Mendoza J, et al. Diagnostic yield and complications of bronchoscopy for peripheral lung lesions. Results of the AQulRE registry. Am J Respir Crit Care Med. 2016;193: 68-77.

33. Bellinger CR, Chatterjee AB, Adair N, Houle T, Khan I, Haponik E. Training in and experience with endobronchial ultrasound. Respiration. 2014;88:478-83.

\section{Submit your next manuscript to BioMed Central} and we will help you at every step:

- We accept pre-submission inquiries

- Our selector tool helps you to find the most relevant journal

- We provide round the clock customer support

- Convenient online submission

- Thorough peer review

- Inclusion in PubMed and all major indexing services

- Maximum visibility for your research

Submit your manuscript at www.biomedcentral.com/submit 